\title{
Sharp moment inequalities for differentially subordinated martingales
}

by

\section{ADAM OsĘKOWski (Warszawa)}

Abstract. We determine the optimal constants $C_{p, q}$ in the moment inequalities

$$
\|g\|_{p} \leq C_{p, q}\|f\|_{q}, \quad 1 \leq p<q<\infty,
$$

where $f=\left(f_{n}\right), g=\left(g_{n}\right)$ are two martingales, adapted to the same filtration, satisfying

$$
\left|d g_{n}\right| \leq\left|d f_{n}\right|, \quad n=0,1,2, \ldots,
$$

with probability 1 . Furthermore, we establish related sharp estimates

$$
\|g\|_{1} \leq \sup _{n} \mathbb{E} \Phi\left(\left|f_{n}\right|\right)+L(\Phi),
$$

where $\Phi$ is an increasing convex function satisfying certain growth conditions and $L(\Phi)$ depends only on $\Phi$.

1. Introduction. The purpose of this paper is to provide new interesting sharp estimates for martingales under the assumption of differential subordination. Let $(\Omega, \mathcal{F}, \mathbb{P})$ be a probability space, filtered by a nondecreasing family $\left(\mathcal{F}_{n}\right)$ of sub- $\sigma$-fields of $\mathcal{F}$. Throughout, $f=\left(f_{n}\right), g=\left(g_{n}\right)$ will stand for martingales adapted to this filtration, taking values in a certain separable Hilbert space $\mathcal{H}$. Their difference sequences $d f=\left(d f_{n}\right), d g=\left(d g_{n}\right)$ are defined by the equations

$$
f_{n}=\sum_{k=0}^{n} d f_{k}, \quad g_{n}=\sum_{k=0}^{n} d g_{k}, \quad n=0,1,2, \ldots
$$

We assume that $g$ is differentially subordinate to $f$, that is, for any $n=$ $0,1,2, \ldots$,

$$
\mathbb{P}\left(\left|d g_{n}\right| \leq\left|d f_{n}\right|\right)=1
$$

For example, this is valid for martingale transforms. Suppose that we have $d g_{n}=v_{n} d f_{n}$ for $n=0,1,2, \ldots$, where $v=\left(v_{n}\right)$ is a real-valued predictable sequence bounded by 1 in absolute value. Obviously, $g$ is then differentially

2010 Mathematics Subject Classification: Primary 60G42; Secondary 60G44.

Key words and phrases: martingale, martingale transform, differential subordination, moment inequality. 
subordinate to $f$. In the special case when each term $v_{n}$ is deterministic and takes values in $\{-1,1\}$, we will say that $g$ is a \pm 1 transform of $f$.

The notion of differential subordination was introduced by Burkholder [Bu2] (though this concept appears in his earlier papers, see e.g. [Bu1]). He also studied various estimates of $g$ in terms of $f$. These include the following weak type $(1,1)$ and moment estimates. Denote $f^{*}=\sup _{n}\left|f_{n}\right|$ and $\|f\|_{p}=\sup _{n}\left\|f_{n}\right\|_{p}$ for $p \in[1, \infty]$.

TheOREm 1.1 (Burkholder). Assume $g$ is differentially subordinate to $f$.

(i) For any $\lambda>0$,

$$
\lambda \mathbb{P}\left(g^{*} \geq \lambda\right) \leq 2\|f\|_{1}
$$

and the constant 2 is the best possible.

(ii) For any $1<p<\infty$,

$$
\|g\|_{p} \leq\left(p^{*}-1\right)\|f\|_{p},
$$

where $p^{*}=\max \{p, p /(p-1)\}$. The constant $p^{*}-1$ is the best possible.

The result above has been extended in many directions. For more information and connections with harmonic analysis, the reader is referred to the survey [Bu4]. For more recent results, see [BW1], [BW2] and [Bu3]- $\mathrm{B}]$ ].

The moment inequality 1.2 fails to hold for $p=1$. However, the following logarithmic estimate is valid. For $K>0$, let $L(K) \in(0, \infty]$ denote the smallest constant $L$ such that the inequality

$$
\|g\|_{1} \leq K \sup _{n} \mathbb{E}\left|f_{n}\right| \log \left|f_{n}\right|+L
$$

holds for all $f, g$ with $g$ being differentially subordinate to $f$.

Here is the main result of [O2].

TheOrem 1.2. We have

$$
L(K)= \begin{cases}\infty & \text { if } 0<K \leq 1, \\ \frac{K^{2}}{2(K-1)} \exp \left(-K^{-1}\right) & \text { if } 1<K<2, \\ K \exp \left(K^{-1}-1\right) & \text { if } K \geq 2 .\end{cases}
$$

In this paper we provide another extension of Theorem 1.1. Namely, for any $p, q$ in $[1, \infty)$ with $p<q$, we will determine the best constants $C_{p, q}$ such that for any martingales $f, g$ with $g$ being differentially subordinate to $f$,

$$
\|g\|_{p} \leq C_{p, q}\|f\|_{q} .
$$

In fact, this estimate will follow from a more general one. We will derive the best constant $L_{p, q}$ such that for any $f, g$ as above,

$$
\|g\|_{p}^{p} \leq\|f\|_{q}^{q}+L_{p, q},
$$

where $1 \leq p<q<2$ or $2<p<q<\infty$. 
The proof of (1.5) depends heavily on the techniques invented by Burkholder. As shown in [Bu4], proving an inequality for differentially subordinate martingales is equivalent to finding a certain special function with convextype properties. To be more specific, let us outline the proof of (1.5). This inequality is equivalent to

$$
\mathbb{E} V_{p, q}\left(f_{n}, g_{n}\right) \leq L_{p, q}, \quad n=0,1,2, \ldots,
$$

where $V_{p, q}: \mathcal{H} \times \mathcal{H} \rightarrow \mathbb{R}$ is given by $V_{p, q}(x, y)=|y|^{p}-|x|^{q}$. We will construct a function $U_{p, q}: \mathcal{H} \times \mathcal{H} \rightarrow \mathbb{R}$ which satisfies

(i) $U_{p, q} \geq V_{p, q}$ on $\mathcal{H} \times \mathcal{H}$,

(ii) $\mathbb{E} U_{p, q}\left(f_{n}, g_{n}\right) \leq \mathbb{E} U_{p, q}\left(f_{n-1}, g_{n-1}\right)$ for $n \geq 1$,

(iii) $U_{p, q}(x, y) \leq L_{p, q}$ if $|y| \leq|x|$.

This will yield the claim: indeed, if $n=0,1,2, \ldots$, then

$$
\mathbb{E} V_{p, q}\left(f_{n}, g_{n}\right) \leq \mathbb{E} U_{p, q}\left(f_{n}, g_{n}\right) \leq \mathbb{E} U_{p, q}\left(f_{0}, g_{0}\right) \leq L_{p, q} .
$$

A few words about the organization of the paper. In the next section we study special solutions to a certain family of differential equations. Then, in Section 3, we introduce two basic functions, which, integrated against special kernels, lead to special functions $U_{p, q}$ corresponding to the moment inequalites 1.5. Section 4 is devoted to the study of the properties of these functions. In the next two sections we establish the moment inequalities (1.4) and (1.5) as well as certain extensions of the estimate $(1.3)$ above. The final section addresses the problem of the strictness and sharpness of these inequalities.

2. A differential equation. As explained above, the inequality 1.5 holds if one manages to find an appropriate majorant $U_{p, q}$ of $V_{p, q}$. How can we construct such a special function? We will present the idea of the search in the case $1<p<2$.

It is natural to try to look at the limit case $p=q$ in the real-valued setting: $\mathcal{H}=\mathbb{R}$. Unfortunately, in that case 1.5$)$ is not valid with any finite $L_{p, p}$ unless $p=2$ (this is clear from Theorem 1.1). However, (1.4) does hold and can be used to gain some insight into the problem. For $x, y \in \mathbb{R}$, let $V_{p, p}(x, y)=|y|^{p}-(p-1)^{-p}|x|^{p}$ and

$$
U_{p, p}(x, y)=p^{2-p}\left(|y|-(p-1)^{-1}|x|\right)(|x|+|y|)^{p-1} .
$$

Then, as shown by Burkholder (see [Bu4]), the functions $U_{p, p}$ and $V_{p, p}$ satisfy conditions (i)-(iii) from the end of the previous section and thus (1.4) holds. Let us take a closer look at the properties of these functions. First, $V_{p, p}(x, y)=U_{p, p}(x, y)$ if and only if $(|x|,|y|)$ lies on the half line $\left\{(\alpha, \beta) \in[0, \infty) \times[0, \infty): \beta=(p-1)^{-1} \alpha\right\}$. Second, condition (ii) is guaranteed by the following geometrical property of $U_{p, p}$ : this function is concave 
along any line of slope $a \in[-1,1]$. Moreover, if we restrict ourselves to the first quadrant, we see that extremal lines (along which $U_{p, p}$ is linear) are those of slope -1 . Finally, $U_{p, p}$ is symmetric: $U_{p, p}(x, y)=U_{p, p}(|x|,|y|)$ and $\left(U_{p, p}\right)_{y}(x, 0)=0$ for all $x$.

It is natural to expect that $U_{p, q}$ should have similar properties. Let us assume that $V_{p, q}(x, y)=U_{p, q}(x, y)$ if and only if $(|x|,|y|)$ lies on a certain curve $\{(\alpha, \beta) \in[0, \infty) \times[0, \infty): \beta=h(\alpha)\}$ and suppose that in the first quadrant $U_{p, q}$ is linear along the lines of slope -1 . These two conditions imply that for $x>0$ and $t \in[-x, h(x)]$,

$$
\begin{aligned}
U_{p, q}(x+t, h(x)-t) & =V_{p, q}(x, h(x))+\left[\left(V_{p, q}\right)_{x}(x, h(x))-\left(V_{p, q}\right)_{y}(x, h(x))\right] t \\
& =h(x)^{q}-x^{p}+\left(q h(x)^{q-1}-p x^{p-1}\right) t .
\end{aligned}
$$

Finally, the condition $\left(U_{p, q}\right)_{y}(x, 0)=0$ leads to the differential equation

$$
p(2-p) h^{\prime}(x)+p=q(q-1) x^{q-2} h(x)^{2-p} .
$$

If we manage to find an appropriate solution, we will obtain an explicit formula for $U_{p, q}$, at least on the set $\{(x, y):|x|+|y| \geq h(0)\}$.

Now we turn to rigorous reasoning. The purpose of this section is to establish, for certain values of $p$ and $q$, the existence of an increasing solution of (2.1) on $[0, \infty)$, satisfying $h(0)>0$ and $h^{\prime}(x) \rightarrow 0, h(x) \rightarrow \infty$, as $x \rightarrow \infty$. As we will see below, if 2 lies between $p$ and $q$, then the constant $C_{p, q}$ is one. Therefore, while studying (1.4), two possibilities are of interest: $1 \leq p<q<2$ or $2<p<q<\infty$. Till the end of Section 4 , we assume that one of them holds.

We consider the cases $1=p<q<2,1<p<q<2$ and $2<p<q<\infty$ separately.

2.1. The case $1=p<q<2$. This is straightforward. There is an explicit formula for the solutions of (2.1), defined on the whole half line $[0, \infty)$. It reads

$$
h(t)=\exp \left(q t^{q-1}\right)\left[\int_{t}^{\infty} \exp \left(-q s^{q-1}\right) d s+\alpha\right], \quad \alpha \in \mathbb{R},
$$

and it is easy to check that the solution $h$ we are interested in corresponds to the case $\alpha=0$.

2.2. Duality. Before we turn to the remaining two cases, let us make a crucial observation. Suppose $p>1$. A deeper look into the equation (2.1) reveals the following dual structure. Let us write $2.1 p_{p, q}$ to indicate the dependence on $p$ and $q$. If $h$ is a solution to $2.1 p, q$, then the function $G$ given by

$$
G(x)=\beta h\left((\alpha x)^{1 /(q-1)}\right)^{p-1},
$$


where

$$
\begin{aligned}
& \alpha=\left(\frac{q}{p}\right)^{p(q-1) /(p-q)}\left(\frac{p-1}{q-1}\right)^{(p-1)(q-1) /(p-q)}, \\
& \beta=\left(\frac{p}{q}\right)^{q(p-1) /(p-q)}\left(\frac{q-1}{p-1}\right)^{(p-1)(q-1) /(p-q)},
\end{aligned}
$$

is a solution to 2.1 $p^{\prime}, q^{\prime}$. Here $p^{\prime}=p /(p-1), q^{\prime}=q /(q-1)$ denote the harmonic conjugates of $p$ and $q$. To see this, note that 2.1) implies

$$
p(2-p) h^{\prime}\left((\alpha x)^{1 /(q-1)}\right)+p=q(q-1)(\alpha x)^{(q-2) /(q-1)} h\left((\alpha x)^{1 /(q-1)}\right)^{2-p} .
$$

Dividing through by the right-hand side, we obtain

$\frac{(2-p) p}{(p-1) q} \frac{1}{\alpha \beta} G^{\prime}(x)+\frac{p}{q(q-1)} \beta^{(2-p) /(p-1)} G(x)^{(p-2) /(p-1)} \cdot(\alpha x)^{(2-q) /(q-1)}=1$

and the choice 2.3 of the parameters $\alpha, \beta$ gives

$$
\left(2-\frac{p}{p-1}\right) G^{\prime}(x)+1=\frac{\frac{q}{q-1} \cdot \frac{1}{q-1} x^{(2-q) /(q-1)} G(x)^{(p-2) /(p-1)}}{p /(p-1)},
$$

i.e. $2.1 p_{p^{\prime}, q^{\prime}}$ for $h=G$.

2.3. The case $1<p<q<2$. We have the following.

THEOREM 2.1. There exists an increasing solution $\hat{h}:[0, \infty) \rightarrow[0, \infty)$ of $2.1 p, q$ satisfying $\hat{h}(0)>0$ and $\hat{h}^{\prime}(t) \rightarrow 0, \hat{h}(t) \rightarrow \infty$, as $t \rightarrow \infty$.

Proof. In fact, we do not know if 2.1$)_{p, q}$ has any solutions on the whole half line $[0, \infty)$. Let $h$ be a function, defined on a certain subinterval of $[0, \infty)$ on which it satisfies the differential equation. This function can be extended to its maximal domain, which is a certain interval $I$. Note that since $(2-p) h^{\prime}(x)+1>0$, the interval $I$ contains its left end; therefore, $I=[a, b],[a, b)$ or $[a, \infty)$. Observe that $a=0$. Indeed, otherwise we would have $h(a)=0$ (by maximality of the domain) and $h^{\prime}(a+)=-1 /(2-p)<0$, a contradiction.

It is convenient to divide the remaining part of the proof into a few steps.

STEP 1. For a fixed $z \in(0, \infty)$, by Picard-Lindelöf's theorem, there is a unique solution $h^{(z)}$ to $2.1 p_{p, q}$, satisfying the condition

$$
q(q-1) z^{q-2}\left[h^{(z)}(z)\right]^{2-p}=p,
$$

or, equivalently, $\left(h^{(z)}\right)^{\prime}(z)=0$ (again, it is extended to its maximal domain). We will show that

$$
\begin{gathered}
h^{(z)} \text { is strictly concave, } \\
h^{(z)}(0)>0
\end{gathered}
$$


Let us write $h$ instead of $h^{(z)}$. We have the identity

$$
h^{\prime \prime}(x)=\frac{q(q-1)}{(2-p) p} h(x)^{1-p} x^{q-3}\left[(2-p) h^{\prime}(x) x-(2-q) h(x)\right] .
$$

Denote the expression in the square brackets by $F(x)$ and derive

$$
F^{\prime}(x)=(2-p) h^{\prime \prime}(x) x+(q-p) h^{\prime}(x) .
$$

Suppose that there is $S \in(0, z)$ for which $h^{\prime \prime}(S)=0$. Then by 2.7) we have $h^{\prime}(S)>0$ and hence $F^{\prime}(S)>0$. It is easy to see that this implies $F^{\prime}(x)>0$ and $h^{\prime \prime}(x)>0$ for $x>S$ and hence $h^{\prime}$ has no zeros larger than $S$, a contradiction. Therefore $h^{\prime \prime}(x) \neq 0$ for $x<z$. However, by (2.7), we have $h^{\prime \prime}(z)<0$ and thus $h^{\prime \prime}(x)<0$ for $x \leq z$. On the other hand, $(2.8)$ gives $F^{\prime}(z)<0$, and so $F^{\prime}(x)<0$ and $h^{\prime \prime}(x)<0$ for $x>z$. This gives 2.5 .

Now note that, by (2.1),

$$
\begin{aligned}
{\left[\frac{p(2-p)}{p-1} h(x)^{p-1}-q x^{q-1}\right]^{\prime} } & =p(2-p) h(x)^{p-2} h^{\prime}(x)-q(q-1) x^{q-2} \\
& =-p h(x)^{p-2}<0
\end{aligned}
$$

and hence

$$
\frac{p(2-p)}{p-1} h(0)^{p-1}>\frac{p(2-p)}{p-1} h(z)^{p-1}-q z^{q-1} .
$$

However, by (2.4), the right-hand side equals

$$
\frac{p(2-p)}{p-1}\left(\frac{p}{q(q-1)}\right)^{(p-1) /(2-p)} z^{(p-1)(2-q) /(2-p)}-q z^{q-1},
$$

which is nonnegative for small $z$, as

$$
\frac{(p-1)(2-q)}{2-p}<q-1 \text {. }
$$

Thus we have proved $h^{(z)}(0)>0$ for small $z$. However, this is sufficient: by (2.4), the greater $z$, the greater $h^{(z)}(z)$, and hence, the greater $h^{(z)}(0)$.

SteP 2. Now we will provide a uniform bound for $h^{(z)}, z \in(0, \infty)$. Let

$$
w=\left(\frac{p(3-q)}{q(q-1)}\right)^{1 /(q-p)}
$$

and denote by $h_{w}$ the unique solution of (2.1) satisfying $h_{w}(w)=w($ extended to its maximal domain). It is easy to check that

$$
(2-p) h_{w}^{\prime}(w) w-(2-q) h_{w}(w)=0
$$

and hence $h_{w}^{\prime \prime}(w)=0$, by (2.7). Thus $h_{w} \neq h^{(z)}$ for any $z$. Furthermore, by (2.9) applied to $h=h_{w}$, we have, for any $x>0$,

$$
\frac{p(2-p)}{p-1} h_{w}(0)^{p-1}>\frac{p(2-p)}{p-1} h_{w}(x)^{p-1}-q x^{q-1},
$$


and hence the maximal domain of $h_{w}$ equals $[0, \infty)$. Now if $h>h_{w}$ is any solution of (2.1), then $h^{\prime}>h_{w}^{\prime}>0$, and therefore $h$ does not coincide with $h^{(z)}$ for any $z$. In other words, $\sup h^{(z)} \leq h_{w}$.

STEP 3. The function we search for in the theorem is defined by

$$
\hat{h}(x)=\sup \left\{h^{(z)}(x): z>x\right\}=\lim _{z \rightarrow \infty} h^{(z)}(x) .
$$

Note that $\hat{h}$ is finite due to Step 3. Clearly, $\hat{h}$ is a solution to 2.1) and $\hat{h}(0)>0$. Furthermore, it is nondecreasing, since $\left(h^{(z)}\right)^{\prime}(x)>0$ for any $x<z$. In fact, it is increasing, since otherwise $h^{\prime}(z)=0$ for some $z$ and thus the function would coincide with $h^{(z)}$, which is impossible. To complete the proof, note that $\hat{h}^{\prime}(x) \leq \hat{h}^{\prime}(1)$ for $x>1$ and, by (2.1),

$$
\hat{h}(x) \leq\left(\frac{p}{q(q-1)}\left((2-p) \hat{h}^{\prime}(1)+1\right) x^{2-q}\right)^{1 /(2-p)},
$$

which implies $\hat{h}^{\prime} \rightarrow 0$ as $t \rightarrow \infty$. Combined with $2.1 p_{p, q}$, this immediately yields $\hat{h}(t) \rightarrow \infty$ as $t \rightarrow \infty$.

2.4. The case $2<p<q<\infty$. Then $q \in(p, \infty)$. We have the following fact.

Theorem 2.2. There exists an increasing solution $\hat{h}:[0, \infty) \rightarrow[0, \infty)$ of 2.1 ${ }_{q, p}$ satisfying $\hat{h}^{\prime}(t) \downarrow 0$ as $t \rightarrow \infty$.

Proof. We use duality. Let $h$ be the solution of $(2.1)_{q^{\prime}, p^{\prime}}$ as in Theorem 2.1 and let $\hat{h}$ be its dual defined by the transformation (2.2). Then $\hat{h}$ is defined on the whole half line $[0, \infty), \hat{h}(0)>0, \hat{h}(t) \rightarrow \infty$ as $t \rightarrow \infty$, and

$$
\hat{h}^{\prime}(x)=\beta \alpha\left(q^{\prime}-1\right)^{-1}\left(x^{\prime}\right)^{2-q^{\prime}} h\left(x^{\prime}\right)^{p^{\prime}-2} h^{\prime}\left(x^{\prime}\right),
$$

where $x^{\prime}=(\alpha x)^{1 /(q-1)}$. It suffices to note that, by $(2.1)_{q^{\prime}, p^{\prime}}$,

$$
\left(x^{\prime}\right)^{2-q^{\prime}} h\left(x^{\prime}\right)^{p^{\prime}-2} \leq \frac{q^{\prime}\left(q^{\prime}-1\right)}{p^{\prime}},
$$

so $h^{\prime}\left(x^{\prime}\right) \rightarrow 0$ as $x \rightarrow \infty$. The proof is complete.

In all the considerations below, we will denote the solution $\hat{h}$ of 2.1 by $h$. We conclude this section by introducing another function to be used later: let $H:[h(0), \infty) \rightarrow[0, \infty)$ be the inverse to $t \mapsto t+h(t)$. We have the following trivial equalities:

$$
\begin{gathered}
h(H(t))+H(t)=t, \\
h^{\prime}(H(t))+1=\frac{1}{H^{\prime}(t)} .
\end{gathered}
$$

Now, as explained at the beginning of this section, we are able to provide a formula for the function $U_{p, q}$. The next step would be to check conditions 
(i)-(iii) listed at the end of Section 1. However, the verification of (ii) is quite elaborate; to overcome this difficulty, we will take a different approach.

3. Simple special functions and the integration argument. In order to prove inequality (1.1), Burkholder invented a special function $W_{1}$ : $\mathcal{H} \times \mathcal{H} \rightarrow \mathbb{R}$ given by

$$
W_{1}(x, y)= \begin{cases}|y|^{2}-|x|^{2} & \text { if }|x|+|y| \leq 1 \\ 1-2|x| & \text { if }|x|+|y|>1\end{cases}
$$

and showed (cf. [Bu4]) that $W_{1}$ has the following property: if $x, y, k_{x}, k_{y} \in \mathcal{H}$ satisfy $\left|k_{y}\right| \leq\left|k_{x}\right|$, then the function $G: \mathbb{R} \rightarrow \mathbb{R}$ given by

$$
G(t)=G_{x, y, k_{x}, k_{y}}(t)=W_{1}\left(x+t k_{x}, y+t k_{y}\right)
$$

is concave. As explained in [Bu4] (see also the proof of Lemma 3.1 below), this property implies that $W_{1}$ satisfies the condition (ii) at the end of Section 1. More specifically, if $f, g$ are martingales such that for some $n \geq 1$ we have $\left|d g_{n}\right| \leq\left|d f_{n}\right|$, then

$$
\mathbb{E} W_{1}\left(f_{n}, g_{n}\right) \leq \mathbb{E} W_{1}\left(f_{n-1}, g_{n-1}\right) .
$$

As a consequence, if $g$ is differentially subordinate to $f$, then the sequence $\left(\mathbb{E} W_{1}\left(f_{n}, g_{n}\right)\right)$ is nonincreasing. This property is preserved by integration against positive kernels. To be more precise, let $w: \mathbb{R}_{+} \rightarrow \mathbb{R}_{+}$be such that for all $x, y \in \mathcal{H}$,

$$
\int_{0}^{\infty} w(t)\left|W_{1}(x / t, y / t)\right| d t<\infty .
$$

Now if we take $\beta \in \mathbb{R}$ and set

$$
U(x, y)=\int_{0}^{\infty} w(t) W_{1}(x / t, y / t) d t+\beta,
$$

then the sequence $\left(\mathbb{E} U\left(f_{n}, g_{n}\right)\right)$ is nonicreasing, provided all the expectations exist. Furthermore, $W_{1}(x, y) \leq 0$ for all $x, y$ satisfying $|y| \leq|x|$, which implies $U(x, y) \leq \beta$ for such $x, y$, and so, for $f$ and $g$ as above,

$$
\mathbb{E} U\left(f_{n}, g_{n}\right) \leq \mathbb{E} U\left(f_{0}, g_{0}\right) \leq \beta, \quad n=0,1,2, \ldots
$$

Using the function $W_{1}$ and integration, we will obtain the special functions corresponding to the moment inequalities in case $1 \leq p<2$. To cover other possible choices of the parameter $p$, we need a dual function to $W_{1}$. Let us introduce $W_{\infty}: \mathcal{H} \times \mathcal{H} \rightarrow \mathbb{R}$ by

$$
W_{\infty}(x, y)= \begin{cases}0 & \text { if }|x|+|y| \leq 1, \\ (|y|-1)^{2}-|x|^{2} & \text { if }|x|+|y|>1 .\end{cases}
$$

The function $W_{\infty}$ has the concavity property described above. More precisely, we have the following. 
Lemma 3.1. Fix $x, y, k_{x}, k_{y} \in \mathcal{H}$ such that $\left|k_{y}\right| \leq\left|k_{x}\right|$. The function $G: \mathbb{R} \rightarrow \mathbb{R}$ given by

$$
G(t)=G_{x, y, k_{x}, k_{y}}(t)=W_{\infty}\left(x+t k_{x}, y+t k_{y}\right)
$$

is concave.

Proof. For $t$ such that $\left|y+t k_{y}\right| \neq 0$ and $\left|x+t k_{x}\right|+\left|y+t k_{y}\right|>1$, we have

$$
G^{\prime \prime}(t)=2\left(\left|k_{y}\right|^{2}-\left|k_{x}\right|^{2}\right)-2 \cdot \frac{\left|k_{y}\right|^{2}-\left(\left(y+t k_{y}\right)^{\prime}, k_{y}\right)^{2}}{|y+t k|} \leq 0,
$$

where we have set $z^{\prime}=z /|z|$ for $z \in \mathcal{H} \backslash\{0\}$. If $\left|x+t k_{x}\right|+\left|y+t k_{y}\right|<1$, then $G^{\prime \prime}(t)=0$. For the remaining $t$ we easily check that $G^{\prime}(t+) \leq G^{\prime}(t-)$.

As previously, this implies the following property: if $g$ is differentially subordinate to $f$ and $f \in L^{2}$, then the sequence $\left(\mathbb{E} W_{\infty}\left(f_{n}, g_{n}\right)\right)$ is nonincreasing. This property carries over to the function $U$ defined by (3.3) with $W_{1}$ replaced by $W_{\infty}$. As $W_{\infty}(x, y) \leq 0$ for $x, y$ satisfying $|y| \leq|x|$, we have, by the arguments used above,

$$
\mathbb{E} U\left(f_{n}, g_{n}\right) \leq \mathbb{E} U\left(f_{0}, g_{0}\right) \leq \beta, \quad n=0,1,2, \ldots
$$

We conclude this section with a result which will be used in the proof of strictness of our main estimates.

LEMMA 3.2. Fix $n \geq 1$ and suppose that $f, g$ are martingales such that $g$ is differentially subordinate to $f$. Assume further that $\left|f_{n-1}\right|+\left|g_{n-1}\right|<1$ almost surely and $\mathbb{P}\left(\left|f_{n}\right|+\left|g_{n}\right|>1\right)>0$. Then

$$
\mathbb{E} W_{1}\left(f_{n}, g_{n}\right)<\mathbb{E} W_{1}\left(f_{n-1}, g_{n-1}\right)
$$

and, if $f$ is square integrable,

$$
\mathbb{E} W_{\infty}\left(f_{n}, g_{n}\right)<\mathbb{E} W_{\infty}\left(f_{n-1}, g_{n-1}\right) .
$$

Proof. Let $x, y, k_{x}, k_{y} \in \mathcal{H}$ satisfy $|x|+|y|<1$ and $\left|k_{y}\right| \leq\left|k_{x}\right|$. The concavity of the function $G$ given by (3.1) gives $G(1) \leq G(0)+G^{\prime}(0)$, or

$$
\begin{aligned}
W_{1}\left(x+k_{x}, y+k_{y}\right) & \leq W_{1}(x, y)+\left(W_{1 x}(x, y), h\right)+\left(W_{1 y}(x, y), k\right) \\
& =|y|^{2}-|x|^{2}-2\left(x, k_{x}\right)+2\left(y, k_{y}\right) .
\end{aligned}
$$

Now, to establish (3.6), it suffices to show that if $\left|x+k_{x}\right|+\left|y+k_{y}\right|>1$, then the above estimate is strict. Indeed, if we prove this, we plug $x=f_{n-1}$, $y=g_{n-1}, k_{x}=d f_{n}$ and $k_{y}=d g_{n}$, thus obtaining an inequality which is strict on a set of positive measure. Taking expectation of both sides yields (3.6). So, assume that $\left|x+k_{x}\right|+\left|y+k_{y}\right|>1$ and observe that the desired estimate can be rewritten in the form

$$
1-2\left|x+k_{x}\right|<\left|y+k_{y}\right|^{2}-\left|x+k_{x}\right|^{2}+\left|k_{x}\right|^{2}-\left|k_{y}\right|^{2},
$$

or

$$
\left(\left|x+k_{x}\right|+\left|y+k_{y}\right|-1\right)\left(\left|x+k_{x}\right|-\left|y+k_{y}\right|-1\right)<\left|k_{x}\right|^{2}-\left|k_{y}\right|^{2} .
$$


If $\left|x+k_{x}\right|<\left|y+k_{y}\right|+1$, then this inequality holds: the left-hand side is negative and the right-hand side is nonnegative. If, conversely, $\left|x+k_{x}\right| \geq$ $\left|y+k_{y}\right|+1$, then, using the trivial bounds $\left|k_{x}\right| \geq|| x+k_{x}|-| x||$ and $\left|k_{y}\right| \leq$ $\left|y+k_{y}\right|+|y|$, we get

$$
\begin{aligned}
\left|k_{x}\right|^{2}-\left|k_{y}\right|^{2} & \geq\left(\left|x+k_{x}\right|+\left|y+k_{y}\right|-|x|+|y|\right)\left(\left|x+k_{x}\right|-\left|y+k_{y}\right|-|x|-|y|\right) \\
& >\left(\left|x+k_{x}\right|+\left|y-k_{y}\right|-1\right)\left(\left|x+k_{x}\right|-\left|y-k_{y}\right|-1\right) .
\end{aligned}
$$

The reasoning for $W_{\infty}$ is similar: we must show that if $x, y, k_{x}, k_{y} \in \mathcal{H}$ satisfy $|x|+|y|<1,\left|x+k_{x}\right|+\left|y+k_{y}\right|>1$ and $\left|k_{y}\right| \leq\left|k_{x}\right|$, then

$$
W_{\infty}\left(x+k_{x}, y+k_{y}\right)<W_{\infty}(x, y)+\left(W_{\infty x}(x, y), k_{x}\right)+\left(W_{\infty y}(x, y), k_{y}\right),
$$

or, equivalently,

$$
\left(\left|x+k_{x}\right|+\left|y+k_{y}\right|-1\right)\left(-\left|x+k_{x}\right|+\left|y+k_{y}\right|-1\right)<0 .
$$

This follows immediately from

$$
-\left|x+k_{x}\right|+\left|y+k_{y}\right|-1 \leq|x|+|y|-1+\left|k_{y}\right|-\left|k_{x}\right|<0 .
$$

4. Special functions. Here we determine the kernels $w$ and numbers $\beta$ which give us the special functions $U_{p, q}: \mathcal{H} \times \mathcal{H} \rightarrow \mathbb{R}$ corresponding to the moment inequality 1.5. For $1 \leq p<q<2$, let $h$ be the solution to 2.1 $p, q$,

$$
w_{p, q}(t)=\frac{p(2-p)}{2} h(H(t))^{p-3} h^{\prime}(H(t)) H^{\prime}(t) t^{2}
$$

and

$$
U_{p, q}(x, y)=\int_{h(0)}^{\infty} w_{p, q}(t) W_{1}(x / t, y / t) d t+\frac{(2-p) h(0)^{p}}{2} .
$$

For $2<p<q<\infty$, let $h$ be the solution to 2.1$)_{q, p}$,

$$
w_{p, q}(t)=\frac{q(q-2)}{2} h(H(t))^{q-3} h^{\prime}(H(t)) H^{\prime}(t) t^{2}
$$

and

$$
\begin{aligned}
U_{p, q}(x, y)= & \int_{h(0)}^{\infty} w_{p, q}(t) W_{\infty}(x / t, y / t) d t \\
& +\frac{q}{2} h(0)^{q-2}\left(|y|^{2}-|x|^{2}\right)+\frac{q-2}{2} h(0)^{q} .
\end{aligned}
$$

Note that the formulas for $w_{p, q}$ in the two cases are essentially the same: one only has to switch the parameters $p$ and $q$ and change the sign.

Let us turn to the explicit formulas for $U_{p, q}$. It can be verified that the formulas in the two lemmas below agree with those introduced at the beginning of Section 2. First we deal with the case $1 \leq p<q<2$. 
Lemma 4.1. Let $1 \leq p<q<2$. We have

$$
U_{p, q}(x, y)=p \frac{|y|^{2}-|x|^{2}}{2 h(0)^{2-p}}+\frac{(2-p) h(0)^{p}}{2}
$$

if $|x|+|y| \leq h(0)$, and

$$
\begin{aligned}
U_{p, q}(x, y)= & p|y| h(H(|x|+|y|))^{p-1}-(p-1) h(H(|x|+|y|))^{p} \\
& -H(|x|+|y|)^{q}-q H(|x|+|y|)^{q-1}(|x|-H(|x|+|y|))
\end{aligned}
$$

if $|x|+|y|>h(0)$.

Proof. Using the formula for $W_{1}$ we see that if $|x|+|y|<h(0)$, then

$$
U_{p, q}(x, y)=\left(|y|^{2}-|x|^{2}\right) \int_{h(0)}^{\infty} \frac{w_{p, q}(t)}{t^{2}} d t+\frac{(2-p) h(0)^{p}}{2} .
$$

Now we have, for any $s>0$,

$$
\int_{s}^{\infty} \frac{w_{p, q}(t)}{t^{2}} d t=\frac{p}{2} \int_{s}^{\infty}\left[-h(H(t))^{p-2}\right]^{\prime} d t=\frac{p}{2} h(H(s))^{p-2}
$$

and hence (4.4) is valid. Suppose now that $|x|+|y| \geq h(0)$. We have

$$
\begin{aligned}
U_{p, q}(x, y)= & \int_{h(0)}^{|x|+|y|} w_{p, q}(t) d t-2|x| \int_{h(0)}^{|x|+|y|} \frac{w_{p, q}(t)}{t} d t \\
& +\left(|y|^{2}-|x|^{2}\right) \int_{|x|+|y|}^{\infty} \frac{w_{p, q}(t)}{t^{2}} d t+\frac{(2-p) h(0)^{p}}{2}
\end{aligned}
$$

and we need to calculate the first and the second integral. First note that as $H$ is inverse to $t \mapsto t+h(t)$, we have

$$
\begin{aligned}
\int_{h(0)}^{s} h(H(t))^{p-2} d t= & \int_{h(0)}^{s} h(H(t))^{p-2} H^{\prime}(t)\left(1+h^{\prime}(H(t))\right) d t \\
= & \int_{h(0)}^{s} h(H(t))^{p-2} H^{\prime}(t) d t \\
& +\int_{h(0)}^{s} h(H(t))^{p-2} h^{\prime}(H(t)) H^{\prime}(t) d t .
\end{aligned}
$$

The equation 2.1), applied to $x=H(t)$, is equivalent to

$h(H(t))^{p-2} H^{\prime}(t)=\frac{q(q-1) H(t)^{q-2} H^{\prime}(t)}{p}+(p-2) h(H(t))^{p-2} h^{\prime}(H(t)) H^{\prime}(t)$, 
which combined with the previous equality gives

$$
\text { 8) } \begin{aligned}
& \int_{h(0)}^{s} h(H(t))^{p-2} d t \\
= & \int_{h(0)}^{s} \frac{q(q-1) H(t)^{q-2} H^{\prime}(t)}{p} d t+(p-1) \int_{h(0)}^{s} h(H(t))^{p-2} h^{\prime}(H(t)) H^{\prime}(t) d t \\
= & \frac{q H(s)^{q-1}}{p}+h(H(s))^{p-1}-h(0)^{p-1} .
\end{aligned}
$$

Therefore, integrating by parts we obtain

$$
\begin{aligned}
\int_{h(0)}^{s} \frac{w_{p, q}(t)}{t} d t & =\frac{p}{2} \int_{h(0)}^{s} t\left[-h(H(t))^{p-2}\right]^{\prime} d t \\
& =-\frac{p}{2} \operatorname{sh}(H(s))^{p-2}+\frac{p}{2} h(0)^{p-1}+\frac{p}{2} \int_{h(0)}^{s} h(H(t))^{p-2} d t \\
& =-\frac{p}{2} \operatorname{sh}(H(s))^{p-2}+\frac{q H(s)^{q-1}}{2}+\frac{p}{2} h(H(s))^{p-1}
\end{aligned}
$$

where in the last passage we have used (4.8). Finally, integration by parts gives

$$
\int_{h(0)}^{s} w_{p, q}(t) d t=\frac{p}{2}\left[-s^{2} h(H(s))^{p-2}+h(0)^{p}\right]+p \int_{h(0)}^{s} t h(H(t))^{p-2} d t
$$

and

$$
\begin{aligned}
\int_{h(0)}^{s} t h(H(t))^{p-2} d t & =\int_{h(0)}^{s}[h(H(t))+H(t)] h(H(t))^{p-2} d t \\
& =\int_{h(0)}^{s} h(H(t))^{p-1} d t+\int_{h(0)}^{s} H(t) h(H(t))^{p-2} d t=I_{1}+I_{2} .
\end{aligned}
$$

Again integrating by parts, we obtain

$$
I_{1}=\operatorname{sh}(H(s))^{p-1}-h(0)^{p}-(p-1) \int_{h(0)}^{s} t h(H(t))^{p-2} h^{\prime}(H(t)) H^{\prime}(t) d t
$$

and, using the equality $t=h(H(t))+H(t)$,

$$
-(p-1) \int_{h(0)}^{s} t h(H(t))^{p-2} h^{\prime}(H(t)) H^{\prime}(t) d t=J_{1}+J_{2}+J_{3},
$$


where

$$
\begin{aligned}
& J_{1}=-(p-1) \int_{h(0)}^{s} h(H(t))^{p-1} h^{\prime}(H(t)) H^{\prime}(t) d t=\frac{p-1}{p}\left[-h(H(s))^{p}+h(0)^{p}\right], \\
& J_{2}=-\int_{h(0)}^{s} h(H(t))^{p-2} h^{\prime}(H(t)) H(t) H^{\prime}(t) d t \\
& J_{3}=(2-p) \int_{h(0)}^{s} h(H(t))^{p-2} h^{\prime}(H(t)) H(t) H^{\prime}(t) d t .
\end{aligned}
$$

We find that $I_{2}+J_{2}$ equals

$$
\int_{h(0)}^{s} h(H(t))^{p-2} H(t)\left[1-h^{\prime}(H(t)) H^{\prime}(t)\right] d t=\int_{h(0)}^{s} h(H(t))^{p-2} H(t) H^{\prime}(t) d t,
$$

so, by (2.1), $I_{2}+J_{2}+J_{3}$ is given by

$$
\begin{aligned}
\frac{1}{p} \int_{h(0)}^{s} q(q-1) H(t)^{q-2} H(t) H^{\prime}(t) d t & =\frac{1}{p} \int_{h(0)}^{s}\left[(q-1) H(t)^{q}\right]^{\prime} d t \\
& =\frac{(q-1) H(s)^{q}}{p} .
\end{aligned}
$$

Summarizing, we have shown that

$$
\begin{aligned}
\int_{h(0)}^{s} w_{p, q}(t) d t= & \frac{p}{2}\left[-s^{2} h(H(s))^{p-2}+h(0)^{p}\right]+p\left(\operatorname{sh}(H(s))^{p-1}-h(0)^{p}\right) \\
& +(p-1)\left(-h(H(s))^{p}+h(0)^{p}\right)+(q-1) H(s)^{q} .
\end{aligned}
$$

Now insert (4.6), 4.9) and 4.10 in 4.7) to obtain 4.5).

Analogous arguments lead to the following formula for $U_{p, q}$ in the case $2<p<q<\infty$.

Lemma 4.2. Let $2<p<q<\infty$. We have

$$
U_{p, q}(x, y)=q \frac{|y|^{2}-|x|^{2}}{2 h(0)^{2-q}}+\frac{q-2}{2} h(0)^{q}
$$

if $|x|+|y| \leq h(0)$, and

$$
\begin{aligned}
U_{p, q}(x, y)= & H(|x|+|y|)^{p}+p H(|x|+|y|)^{p-1}(|y|-H(|x|+|y|)) \\
& -h(H(|x|+|y|))^{q}+q h(H(|x|+|y|))^{q-1}(|y|-H(|x|+|y|))
\end{aligned}
$$

if $|x|+|y|>h(0)$. 
Proof. If $|x|+|y| \leq h(0)$, then the integral term in the definition of $U_{p, q}$ vanishes and 4.11) is valid. If $|x|+|y|>h(0)$, then

$$
\begin{aligned}
U_{p, q}(x, y)= & \left(|y|^{2}-|x|^{2}\right) \int_{h(0)}^{|x|+|y|} \frac{w_{p, q}(t)}{t^{2}} d t-2|y| \int_{h(0)}^{|x|+|y|} \frac{w_{p, q}(t)}{t} d t \\
& +\int_{h(0)}^{|x|+|y|} w_{p, q}(t) d t+q \frac{|y|^{2}-|x|^{2}}{2 h(0)^{2}-q}+\frac{q-2}{2} h(0)^{q}
\end{aligned}
$$

and the first integral is given by

$$
\int_{h(0)}^{|x|+|y|}\left(\frac{q}{2} h(H(t))^{q-2}\right)^{\prime} d t=\frac{q}{2}\left(h(H(|x|+|y|))^{q-2}-h(0)^{q-2}\right) .
$$

To compute the remaining two integrals, we recall the similarity of the formulas for $w_{p, q}$ in the cases $1 \leq p<q<2,2<p<q<\infty$ and see that the arguments leading to 4.9 and (4.10) are still valid (one only needs to interchange $p$ and $q$, and change the sign). Now insert the equalities to 4.13 to obtain 4.12.

Now we turn to the majorization property. Recall that $V_{p, q}: \mathcal{H} \times \mathcal{H} \rightarrow \mathbb{R}$ is given by $V_{p, q}(x, y)=|y|^{p}-|x|^{q}$.

Lemma 4.3. For any $(x, y) \in \mathcal{H} \times \mathcal{H}$ we have $U_{p, q}(x, y) \geq V_{p, q}(x, y)$.

Proof. Clearly, it suffices to prove the lemma for $\mathcal{H}=\mathbb{R}$. We will present all the details in the case $1 \leq p<q<2$ only; the remaining case can be established in the same manner. For the convenience of the reader, the proof is split into a few parts.

STEP 1: Reduction to the set $|x|+|y| \geq h(0)$. If $|x|+|y|<h(0)$, then, for fixed $x$, the expression $U_{p, q}(x, y)-V_{p, q}(x, y)$, considered as a function of $|y|$, is nonincreasing (simply calculate the derivative and use the fact that $|y| \leq h(0))$. Hence $U_{p, q}(x, y)-V_{p, q}(x, y) \geq U_{p, q}(x, z)-V_{p, q}(x, z)$, where $z \in \mathcal{H}$ satisfies $|x|+|z|=h(0)$. Therefore, it suffices to establish the majorization on the set $|x|+|y| \geq h(0)$.

STEP 2: $p=1$. The majorization follows from the convexity of the function $t \mapsto t^{q}$ : indeed, the inequality $U_{p, q}(x, y) \geq V_{p, q}(x, y)$ is equivalent to

$$
|x|^{q}-H(|x|+|y|)^{q}-q H(|x|+|y|)^{q-1} \cdot(|x|-H(|x|+|y|)) \geq 0 .
$$

Therefore, till the end of the proof, we assume that $p \neq 1$.

SteP 3: Reduction to the case $y=0$. Fix $r \geq h(0)$ and suppose $|x|+|y|=r$. Denoting $s=|y|$ we see that $U_{p, q}(x, y) \geq V_{p, q}(x, y)$ is equivalent to 


$$
\begin{aligned}
F(s)= & p s h(H(r))^{p-1}-(p-1) h(H(r))^{p} \\
& -H(r)^{q}-q H(r)^{q-1} h(H(r))-s^{p}+(r-s)^{q} \geq 0 .
\end{aligned}
$$

We have $F(h(H(r)))=F^{\prime}(h(H(r)))=0$. Furthermore, the second derivative of $F$, equal to $F^{\prime \prime}(s)=-p(p-1) s^{p-2}+q(q-1)(r-s)^{q-2}$, is negative on $\left(0, s_{0}\right)$ and positive on $\left(s_{0}, r\right)$ for some $s_{0} \in(0, r)$. Therefore, to show (4.14), it suffices to prove that $F(0) \geq 0$, or $U_{p, q}(x, 0) \geq V_{p, q}(x, 0)$.

STEP 4: $U_{p, q}(x, 0)>V_{p, q}(x, 0)$ for large $|x|$. As $q<2$, we have, for large $s$,

$$
\begin{aligned}
\frac{s^{q}-(H(s))^{q}-q(H(s))^{q-1} h(H(s))}{h(H(s))^{p}}>\frac{q(q-1)}{2} s^{q-2} h(H(s))^{2-p} \\
=\frac{q(q-1)}{2} H(s)^{q-2} h(H(s))^{2-p} \cdot\left(\frac{s}{H(s)}\right)^{q-2} .
\end{aligned}
$$

But, by (2.1),

$$
\frac{q(q-1)}{2} H(s)^{q-2} h(H(s))^{2-p} \geq \frac{p}{2}
$$

and, by 2.10 and Theorem 2.1.

$$
\frac{s}{H(s)}=1+\frac{h(H(s))}{H(s)} \rightarrow 1 \quad \text { as } s \rightarrow \infty .
$$

Since $p / 2>p-1$, we see that

$$
\frac{s^{q}-H(s)^{q}-q H(s)^{q-1} h(H(s))}{h(H(s))^{p}}>p-1
$$

for large $s$. This is equivalent to $U_{p, q}(x, 0)>V_{p, q}(x, 0)$ with $|x|=s$.

SteP 5: $U_{p, q}(x, 0) \geq V_{p, q}(x, 0)$ : the general case. Suppose the inequality does not hold for some $x$. Let $T$ denote the largest $t$ satisfying $U_{p, q}(t, 0)=$ $V_{p, q}(t, 0)$ (its existence is guaranteed by the continuity of $U_{p, q}$ and $V_{p, q}$ and the previous step). By the considerations above, $U_{p, q} \geq V_{p, q}$ on the set $|x|+|y| \geq T$. Consider the processes $f=\left(f_{0}, f_{1}, f_{2}\right), g=\left(g_{0}, g_{1}, g_{2}\right)$ on a probability space $([0,1], \mathcal{B}([0,1]),|\cdot|)$ such that $\left(f_{0}, g_{0}\right) \equiv(T, 0)$ and

$$
\begin{aligned}
& d f_{1}=d g_{1}=\delta \chi_{\left[0, t_{0}\right]}-h(H(T)) \chi_{\left(t_{0}, 1\right]}, \\
& d f_{2}=-d g_{2}=\delta \chi_{\left[0, t_{1}\right]}-(h(H(T))-\delta) \chi_{\left(t_{1}, t_{0}\right]},
\end{aligned}
$$

where

$$
t_{0}=\frac{h(H(T))}{h(H(T))+\delta}, \quad t_{1}=t_{0} \cdot \frac{h(H(T))-\delta}{h(H(T))}=\frac{h(H(T))-\delta}{h(H(T))+\delta} .
$$

It is straightforward to check that $f$ and $g$ are martingales and $g$ is differentially subordinate to $f$. Note that $U_{p, q}\left(f_{2}, g_{2}\right) \geq V_{p, q}\left(f_{2}, g_{2}\right)$ almost surely, 
as $\left|f_{2}\right|+\left|g_{2}\right| \geq T$ with probability 1 . By 3.2 with $W$ replaced by $U_{p, q}$,

$$
\begin{aligned}
-T^{q} & =U_{p, q}(T, 0)=\mathbb{E} U_{p, q}\left(f_{0}, g_{0}\right) \geq \mathbb{E} U_{p, q}\left(f_{2}, g_{2}\right) \geq \mathbb{E} V_{p, q}\left(f_{2}, g_{2}\right) \\
& =\frac{2 \delta}{h(H(T))+\delta}\left(h(H(T))^{p}-H(T)^{q}\right)-\frac{h(H(T))-\delta}{h(H(T))+\delta}(T+2 \delta)^{q},
\end{aligned}
$$

which is equivalent to

$$
\frac{(T+2 \delta)^{q}-T^{q}}{2 \delta}-\frac{(T+2 \delta)^{q}}{h(H(T))+\delta}+\frac{H(T)^{q}}{h(H(T))+\delta} \geq \frac{h(H(T))^{p}}{h(H(T))+\delta} .
$$

Letting $\delta \rightarrow 0$ and multiplying through by $h(H(T))$ yields

$$
h(H(T))^{p} \leq H(T)^{q}-T^{q}-q T^{q-1} h(H(T)) .
$$

But

$$
H(T)^{q}-T^{q}-q T^{q-1} h(H(T)) \leq T^{q}-H(T)^{q}-q H(T)^{q-1} h(H(T)) .
$$

To see this, substitute $H(T)=a>0, T-H(T)=b>0$ and observe that (4.16) becomes

$$
2\left[(a+b)^{q}-a^{q}\right]-\left[q a^{q-1}+q(a+b)^{q-1}\right] b \geq 0 .
$$

Now calculate the derivative of the left-hand side with respect to $b$ : it is equal to

$$
q(a+b)^{q-1}-q a^{q-1}-q(q-1)(a+b)^{q-2} b \geq 0,
$$

due to the mean value theorem. Thus (4.16) follows.

To conclude the proof, observe that (4.15) and (4.16) give

$$
h(H(T))^{p} \leq T^{q}-H(T)^{q}-q H(T)^{q-1} h(H(T)),
$$

and since $p<2$, this implies

$$
(p-1) h(H(T))^{p}<T^{q}-H(T)^{q}-q H(T)^{q-1} h(H(T)),
$$

a contradiction: this inequality is equivalent to $U_{p, q}(T, 0)<V_{p, q}(T, 0)$.

REMARK 4.4. A careful inspection of the proof shows that $U_{p, q}(x, y)=$ $V_{p, q}(x, y)$ if and only if $|y|=h(|x|)$.

5. Proofs of moment inequalities. Now we are ready to establish the inequalities announced in the introduction. Suppose $1 \leq p<q<\infty$ and let

$$
L_{p, q}= \begin{cases}\frac{1}{2}(2-p) h(0)^{p} & \text { if } 1 \leq p<q<2, \\ \frac{1}{2}(q-2) h(0)^{q} & \text { if } 2<p<q<\infty\end{cases}
$$

and

$$
C_{p, q}= \begin{cases}L_{p, q}^{(q-p) / p q}\left(\frac{q-p}{p}\right)^{1 / q}\left(\frac{q}{q-p}\right)^{1 / p} & \text { if } 1 \leq p<q<2, \\ & \text { or } 2<p<q<\infty \\ 1 & \text { otherwise. }\end{cases}
$$


TheOrem 5.1. Assume $g$ is differentially subordinate to $f$.

(i) If $1 \leq p<q<2$ or $2<p<q<\infty$, then (1.5) holds.

(ii) If $1 \leq p<q<\infty$, then the inequality (1.4) holds.

REMARK 5.2. (i) If $p=1$, then there are explicit formulas for the constants $C_{p, q}$, given by

$$
C_{1, q}= \begin{cases}{\left[\frac{q}{2} \Gamma\left(\frac{q}{q-1}\right)\right]^{(q-1) / q}} & \text { if } q \in(1,2), \\ 1 & \text { if } q \geq 2 .\end{cases}
$$

(ii) It is easy to check that $C_{p, q}=C_{q^{\prime}, p^{\prime}}$ for $1<p<q<\infty$.

Proof of Theorem 5.1. With no loss of generality we may assume $0<$ $\|f\|_{q}<\infty$, which, by Burkholder's inequality (1.2), implies $\|g\|_{p} \leq\|g\|_{q}<\infty$. Since $H(s) \leq s$ and $h(H(s)) \leq s$, it follows that the variables $U_{p, q}\left(f_{n}, g_{n}\right)$, $n=0,1,2, \ldots$, are integrable and, by Lemma 4.3 and $(3.4)$, for any $n=$ $0,1,2, \ldots$,

$$
\mathbb{E} V_{p, q}\left(f_{n}, g_{n}\right) \leq \mathbb{E} U_{p, q}\left(f_{n}, g_{n}\right) \leq \mathbb{E} U_{p, q}\left(f_{0}, g_{0}\right) \leq L_{p, q} .
$$

It suffices to let $n \rightarrow \infty$ to obtain (1.5). Now we turn to (1.4). If $p \leq 2 \leq q$, the inequality is clear: we have $\|g\|_{p} \leq\|g\|_{2} \leq\|f\|_{2} \leq\|f\|_{q}$. Suppose that $1 \leq p<q<2$. As already proved, we have

$$
\|g\|_{p}^{p} \leq\|f\|_{q}^{q}+L_{p, q} .
$$

Since for $\lambda>0, g \cdot \lambda^{1 /(q-p)}$ is differentially subordinate to $f \cdot \lambda^{1 /(q-p)}$, we obtain

$$
\|g\|_{p}^{p} \leq \lambda\|f\|_{q}^{q}+\frac{L_{p, q}}{\lambda^{p /(q-p)}} .
$$

It can be easily checked that the right-hand side, as a function of $\lambda$, attains its minimum for

$$
\lambda=\left(\frac{p}{q-p} \cdot \frac{L_{p, q}}{\|f\|_{q}^{q}}\right)^{(q-p) / q}
$$

and the minimum is equal to

$$
\left[L_{p, q}^{(q-p) / p q} \cdot\left(\frac{q-p}{p}\right)^{1 / q}\left(\frac{q}{q-p}\right)^{1 / p}\|f\|_{q}\right]^{p} .
$$

The case $2<p<q<\infty$ is dealt with in exactly the same manner.

Theorem 5.3. Let $1<p<\infty$. Then

$$
\lim _{p^{\prime}, q^{\prime} \rightarrow p} C_{p^{\prime}, q^{\prime}}=p^{*}-1 .
$$

Proof. The inequalities (1.2) and (1.4) are sharp even if $\mathcal{H}=\mathbb{R}, f$ is simple and $g$ is a \pm 1 transform of $f$ (see $[\mathrm{Bu} 2]$ and Theorem 7.2 below). Recall that $f$ is simple if for any $n$ the variable $f_{n}$ takes only a finite number 
of values and there is a deterministic $N$ such that $f_{N}=f_{N+1}=f_{N+2}=\cdots$ with probability 1 . Note that if $f$ is simple, then so is its \pm 1 transform $g$.

Fix $1<p<\infty$ and a pair $(f, g)$ as above. Let $1 \leq p_{n}<q_{n}<\infty$, $n=0,1,2, \ldots$, satisfy $\lim _{n \rightarrow \infty} p_{n}=\lim _{n \rightarrow \infty} q_{n}=p$. Observe that we have $\|g\|_{p_{n}} \leq C_{p_{n}, q_{n}}\|f\|_{q_{n}}, \lim _{n \rightarrow \infty}\|g\|_{p_{n}}=\|g\|_{p}$ and $\lim _{n \rightarrow \infty}\|f\|_{q_{n}}=\|f\|_{p}$ (there are no problems with integrability, as the martingales are simple). Consequently, $\|g\|_{p} \leq \liminf _{n \rightarrow \infty} C_{p_{n}, q_{n}}\|f\|_{p}$ and hence

$$
p^{*}-1 \leq \liminf _{n \rightarrow \infty} C_{p_{n}, q_{n}} .
$$

On the other hand, for any $n$ we have $\|g\|_{p_{n}} \leq\|g\|_{q_{n}} \leq\left(q_{n}^{*}-1\right)\|f\|_{q_{n}}$, which implies $C_{p_{n}, q_{n}} \leq q_{n}^{*}-1$ and

$$
\limsup _{n \rightarrow \infty} C_{p_{n}, q_{n}} \leq p^{*}-1 .
$$

6. Related estimates for the first moment. The method above can be extended to establish some related inequalities. Suppose $\Phi:[0, \infty) \rightarrow$ $[0, \infty)$ is a function satisfying the following conditions: $\Phi$ is an increasing convex $C^{2}$ function such that $\Phi(0)=\Phi^{\prime}(0+)=0$,

$$
\Phi^{\prime \prime}(t) \downarrow 0 \quad \text { as } t \rightarrow \infty
$$

and

$$
\int_{0}^{\infty} \exp \left(-\Phi^{\prime}(s)\right) d s<\infty .
$$

For example, one can take $\Phi(t)=t^{q}, q \in(1,2)$, or $\Phi(t)=t \log (1+t)$. Note that the above conditions imply that $\Phi$ is strictly convex.

The purpose of this section is to establish the inequality

$$
\|g\|_{1} \leq \sup _{n} \mathbb{E} \Phi\left(\left|f_{n}\right|\right)+L(\Phi)
$$

for any pair $(f, g)$ of $\mathcal{H}$-valued martingales such that $g$ is differentially subordinate to $f$. Here $L(\Phi)$ is a constant which depends only on $\Phi$. Let

$$
h(t)=\exp \left(\Phi^{\prime}(t)\right) \int_{t}^{\infty} \exp \left(-\Phi^{\prime}(s)\right) d s
$$

and let $H$ be an inverse function to $t \mapsto t+h(t)$. The special function $U_{1, \Phi}$ : $\mathcal{H} \times \mathcal{H} \rightarrow \mathbb{R}$ corresponding to 6.3 is defined as follows: if $|x|+|y| \leq h(0)$, then

$$
U_{1, \Phi}(x, y)=\frac{|y|^{2}-|x|^{2}}{2 h(0)}+\frac{h(0)}{2}
$$

while for $|x|+|y|>h(0)$ and $|y| \leq h(|x|)$, set

$$
U_{1, \Phi}(x, y)=|y|-\Phi(H(|x|+|y|))-\Phi^{\prime}(H(|x|+|y|))(|x|-H(|x|+|y|)) .
$$


Finally, if $|y|>h(|x|)$ (then $|x|+|y|>h(0))$, define

$$
U_{1, \Phi}(x, y)=|y|-\Phi(|x|) \text {. }
$$

If one takes $\Phi(t)=t^{q}, 1<q<2$, one obtains an alternative special function for the moment inequalities. One can check (arguing as in the proof of Lemma 4.1) that we have the following integral identity: for $|y| \leq h(|x|)$,

$$
U_{1, \Phi}(x, y)=\int_{h(0)}^{\infty} w_{1, \Phi}(t) W_{1}(x / t, y / t) d t
$$

where

$$
w_{1, \Phi}(t)=\frac{1}{2} h(H(t))^{-2} h^{\prime}(H(t)) H^{\prime}(t) t^{2} .
$$

The reason for which we modify the function on the set $|y|>h(|x|)$ (and use the formula (6.7) instead of (6.6) ) is to avoid the problem of integrability of the variables $U_{1, \Phi}\left(f_{n}, g_{n}\right), n=0,1,2, \ldots$

The key properties of the function $U_{1, \Phi}$ are gathered in the lemma below. The function $V_{1, \Phi}: \mathcal{H} \times \mathcal{H} \rightarrow \mathbb{R}$ is given by $V_{1, \Phi}(x, y)=|y|-\Phi(|x|)$.

LEMMA 6.1.

(i) For any $(x, y) \in \mathcal{H} \times \mathcal{H}$ we have $U_{1, \Phi}(x, y) \geq V_{1, \Phi}(x, y)$. Equality holds if and only if $|y| \geq h(|x|)$.

(ii) Assume that $x, y, k_{x}, k_{y} \in \mathcal{H}$ satisfy $\left|k_{y}\right| \leq\left|k_{x}\right|$. Then the function $G: \mathbb{R} \rightarrow \mathbb{R}$ given by $G(t)=U_{1, \Phi}\left(x+t k_{x}, y+t k_{y}\right)$ is concave.

Proof. (i) Arguing as in the proof of Lemma 4.3, we see that it suffices to establish the majorization for $|x|+|y|>h(0)$. If $|y| \geq h(|x|)$, then we have equality: $U_{1, \Phi}(x, y)=V_{1, \Phi}(x, y)$. For remaining $x, y$, the estimate is equivalent to

$$
\Phi(|x|)-\Phi(H(|x|+|y|))-\Phi^{\prime}(H(|x|+|y|))(|x|-H(|x|+|y|)) \geq 0,
$$

which follows from the convexity of $\Phi$. In fact, the latter inequality is strict, since $\Phi$ is strictly convex.

(ii) By (6.8), the function $G$ is concave on the set of those $t$ for which $\left|y+t k_{y}\right|<h\left(\left|x+t k_{x}\right|\right)$. Let us prove $G^{\prime \prime}(t)<0$ for $t$ such that $\left|y+t k_{y}\right|>$ $h\left(\left|x+t k_{x}\right|\right),\left|x+t k_{x}\right|>0$. By translation, we may assume $t=0$. We have

$$
G^{\prime \prime}(0)=\frac{\left|k_{y}\right|^{2}-\left(y^{\prime}, k_{y}\right)^{2}}{|y|}-\Phi^{\prime \prime}(|x|)\left(x^{\prime}, k_{x}\right)^{2}-\Phi^{\prime}(|x|) \frac{\left|k_{x}\right|^{2}-\left(x^{\prime}, k_{x}\right)^{2}}{|x|},
$$

which, as $\Phi^{\prime}(|x|) \geq \Phi^{\prime \prime}(|x|)|x|$, can be bounded from above by

$$
\begin{aligned}
\frac{\left|k_{y}\right|^{2}}{|y|}-\Phi^{\prime \prime}(|x|)\left|k_{x}\right|^{2} & \leq \frac{\left|k_{y}\right|^{2}}{h(|x|)}-\Phi^{\prime \prime}(|x|)\left|k_{x}\right|^{2} \\
& =\frac{\left|k_{y}\right|^{2}-\left|k_{x}\right|^{2}}{h(|x|)}+\frac{\left(1-\Phi^{\prime \prime}(|x|) h(|x|)\right)\left|k_{x}\right|^{2}}{h(|x|)} .
\end{aligned}
$$


It suffices to show that both summands appearing in the last expression are nonpositive. This is clear for the first one, as $\left|k_{y}\right| \leq\left|k_{x}\right|$. To deal with the second one, note that by concavity of $\Phi^{\prime}$, for any $s>t$ we have

$$
\Phi^{\prime}(s) \leq \Phi^{\prime}(t)+\Phi^{\prime \prime}(t)(s-t)
$$

and hence, for any $t$,

$$
h(t)=\int_{t}^{\infty} \exp \left(\Phi^{\prime}(t)-\Phi^{\prime}(s)\right) d s \geq \int_{t}^{\infty} \exp \left(-\Phi^{\prime \prime}(t)(s-t)\right) d s=\frac{1}{\Phi^{\prime \prime}(t)} .
$$

The final observation is that $G^{\prime}(t+) \leq G^{\prime}(t-)$ if $\left|y+t k_{y}\right|=h\left(\left|x+t k_{x}\right|\right)$ or $\left|x+t k_{x}\right|=0$; hence $G$ is concave.

Now we can prove the following result.

Theorem 6.2. Assume $g$ is differentially subordinate to $f$. Then the inequality 6.3 holds with

$$
L(\Phi)=\frac{h(0)}{2}=\frac{1}{2} \int_{0}^{\infty} \exp \left(-\Phi^{\prime}(s)\right) d s .
$$

Proof. We proceed as previously. With no loss of generality we may assume that $\sup _{n} \mathbb{E} \Phi\left(\left|f_{n}\right|\right)<\infty$, which guarantees the integrability of $U_{1, \Phi}\left(f_{n}, g_{n}\right), n=0,1,2, \ldots$, and we have

$$
\mathbb{E} V_{1, \Phi}\left(f_{n}, g_{n}\right) \leq \mathbb{E} U_{1, \Phi}\left(f_{n}, g_{n}\right) \leq \mathbb{E} U_{1, \Phi}\left(f_{0}, g_{0}\right) \leq L(\Phi)
$$

for all $n$. Let $n \rightarrow \infty$ to complete the proof.

As an example, we will establish a family of $\log \log$-estimates for the first moment of the martingale $g$. As a motivation, note that the inequality (1.3) holds with some finite $L$ if and only if $K>1$. Therefore, there is a natural question what happens in the limit case $K=1$. The (partial) answer is contained in the following theorem.

TheOREM 6.3. If $g$ is differentially subordinate to $f$, then, for any $K>1$,

$$
\begin{aligned}
& \|g\|_{1} \leq \sup _{n}\left[\mathbb{E}\left|f_{n}\right| \log \left(\left|f_{n}\right|+1\right)+K \mathbb{E} \log \log \left(\left|f_{n}\right|+e\right)\right] \\
& +\frac{1}{2} \int_{0}^{\infty} \frac{1}{(s+1)(\log (s+e))^{K}} \exp \left(-\frac{s}{s+1}-\frac{K s}{(s+e) \log (s+e)}\right) d s .
\end{aligned}
$$

To prove this, let $\Phi$ be given by $\Phi(t)=t \log (t+1)+K t \log \log (t+e)$. Then the assertion follows from Theorem 6.2, once we check that this function has all the properties given at the beginning of this section. Clearly, $\Phi$ is convex, $C^{2}$ and $\Phi(0)=\Phi^{\prime}(0+)=0$. Furthermore,

Lemma 6.4. For any $K>0$ the condition (6.1) is valid. 
Proof. It is easy to see that $\Phi^{\prime \prime}(t) \rightarrow 0$ as $t \rightarrow \infty$. Hence it suffices to show that $\Phi^{\prime \prime \prime}<0$. Let $\psi_{1}=t \log (t+1)$ and $\psi_{2}(t)=t \log \log (t+e)$. We have

$$
\psi_{1}^{\prime \prime \prime}(t)=-\frac{2}{(t+1)^{3}}-\frac{1}{(t+1)^{2}}<0
$$

and

$$
\psi_{2}^{\prime \prime \prime}(t)=\frac{-3 e \log (t+e)-3 e \log ^{2}(t+e)-t \log ^{2}(t+e)+2 t}{((t+e) \log (t+e))^{3}} .
$$

If $t \in(0,3 e]$, then $2 t \leq 6 e \leq 3 e \log (t+e)+3 e \log ^{2}(t+e)$, which implies that the derivative is negative. This is also true for $t>3 e$, which follows from the estimate $2 t<t \log ^{2}(4 e)<t \log ^{2}(t+e)$.

The last property we need is the following.

Lemma 6.5. If $K>1$, then the condition (6.2) holds.

Proof. We have

$$
\begin{aligned}
\Phi^{\prime}(t) & =\log (t+1)+K \log \log (t+e)+\frac{t}{t+1}+K \frac{t}{(t+e) \log (t+e)} \\
& \geq \log (t+1)+K \log \log (t+e) .
\end{aligned}
$$

Therefore

$$
\int_{0}^{\infty} \exp \left(-\Phi^{\prime}(s)\right) d s<\int_{0}^{\infty} \frac{d s}{(s+1)(\log (s+e))^{K}}<\infty .
$$

Therefore we may apply Theorem 6.2 to the function $\Phi$. One easily checks that the constant $L(\Phi)$ is given by the expression appearing on the right-hand side of (6.11).

7. Strictness and sharpness. In this final section of the paper, we address the strictness of the estimates studied above as well as the optimality of the constants $L_{p, q}, C_{p, q}$ and $L(\Phi)$. First we establish the following result.

THEOREM 7.1. The inequality 1.5 is strict for all $1 \leq p<q<2$ and $2<p<q<\infty$, unless both sides are infinite. The inequality (1.4) is strict for $1 \leq p<q<2$ and $2<p<q<\infty$, unless both sides are infinite or equal to 0 . If $2 \in[p, q]$, then both sides of (1.4) may be equal for some nontrivial $f$ and $g$. The inequality (6.3) is strict unless both sides are infinite.

Proof. We start by showing that equality in 1.5 cannot be attained. First we consider the case $1 \leq p<q<2$. Let $h$ be the corresponding solution to 2.1 $p, q$ and fix martingales $f, g$ such that $\|f\|_{q}<\infty$ and $g$ is differentially subordinate to $f$. Our goal is to prove that $\|g\|_{p}<\|f\|_{q}+$ $L_{p, q}$. We may assume that $\left(f_{0}, g_{0}\right) \equiv(0,0)$, which can be shown by the following standard argument. Take a Rademacher variable $\varepsilon$, independent of $\sigma\left(\bigcup_{n=1}^{\infty} \mathcal{F}_{n}\right)$ (enlarging the probability space, if necessary) and replace $(f, g)$ 
by the pair $(\hat{f}, \hat{g})$ given by $\left(\hat{f}_{0}, \hat{g}_{0}\right)=(0,0)$ and $\hat{f}_{n}=\varepsilon f_{n-1}, \hat{g}_{n}=\varepsilon g_{n-1}$, relative to the filtration $\{\emptyset, \Omega\} \subset \sigma\left(\mathcal{F}_{0}, \varepsilon\right) \subset \sigma\left(\mathcal{F}_{1}, \varepsilon\right) \subset \cdots$. Then $\hat{f}, \hat{g}$ have the required initial property and $\|\hat{f}\|_{q}=\|f\|_{q},\|\hat{g}\|_{p}=\|g\|_{p}$.

We will consider two cases: first, assume that that there is an integer $N$ such that $\mathbb{P}\left(\left|f_{N}\right|+\left|g_{N}\right|>h(0)\right)>0$ and pick the smallest such $N$. Note that $N \geq 1$, since $f$ and $g$ start from 0 . Then, for $t$ in some interval of the form $(h(0), T)$, we have $\left|f_{N-1} / t\right|+\left|g_{N-1} / t\right|<1$ almost surely and $\mathbb{P}\left(\left|f_{N} / t\right|+\right.$ $\left.\left|g_{N} / t\right|>1\right)>0$. By Lemma 3.1, for these $t$,

$$
\mathbb{E} W_{1}\left(f_{N} / t, g_{N} / t\right)<\mathbb{E} W_{1}\left(f_{N-1} / t, g_{N-1} / t\right) .
$$

Since the kernel $w_{p, q}$ defined in 4.1) is positive, we obtain, by (4.2),

$$
\mathbb{E} U_{p, q}\left(f_{N}, g_{N}\right)<\mathbb{E} U_{p, q}\left(f_{N-1}, g_{N-1}\right) .
$$

This implies the strictness:

$$
\|g\|_{p}^{p}-\|f\|_{q}^{q}=\lim _{n \rightarrow \infty} \mathbb{E} V_{p, q}\left(f_{n}, g_{n}\right) \leq \mathbb{E} U_{p, q}\left(f_{N}, g_{N}\right)<\mathbb{E} U_{p, q}\left(f_{0}, g_{0}\right)=L_{p, q} .
$$

We turn to the second case when for all $n$ we have $\left|f_{n}\right|+\left|g_{n}\right| \leq h(0)$ almost surely. Then the martingales $f, g$ are bounded, so in particular they converge in $L^{2}$ to, say, $f_{\infty}$ and $g_{\infty}$. We have $\left|f_{\infty}\right|+\left|g_{\infty}\right| \leq h(0)$ almost surely and, by (1.2), $\left\|g_{\infty}\right\|_{2}^{2} \leq\left\|f_{\infty}\right\|_{2}^{2}$, so

$$
\mathbb{P}\left(\left|f_{\infty}\right|>0\right)>0 \quad \text { or } \quad\left|f_{\infty}\right|=\left|g_{\infty}\right|=0 .
$$

This implies

$$
\|g\|_{p}^{p}-\|f\|_{q}^{q}=\left\|g_{\infty}\right\|_{p}^{p}-\left\|f_{\infty}\right\|_{q}^{q}=\mathbb{E} V_{p, q}\left(f_{\infty}, g_{\infty}\right)<\mathbb{E} U_{p, q}\left(f_{\infty}, g_{\infty}\right) \leq L_{p, q},
$$

where the strict inequality comes from Remark 4.4 and (7.1): the only $x, y$ satisfying $|x|+|y| \leq h(0)$ and $|y|=h(|x|)$ are given by $x=0$ and $|y|=h(0)$. This shows that there are no $f, g$ with $\|f\|_{q}<\infty$ for which both sides of (1.5) are equal. The case $2<p<q<\infty$ can be studied in the same manner, using the second inequality from Lemma 3.1. Clearly, this implies that (1.4) is strict for $1 \leq p<q<2$ and $2<p<q<\infty$, unless $\|f\|_{q}=\|g\|_{p}=0$ or $\|f\|_{q}=\|g\|_{p}=\infty$. On the other hand, if $2 \in[p, q]$, then equality in (1.4) can be attained: take, for example, $f=g \equiv 1$.

We conclude by observing that (6.3) is also strict unless both sides are infinite. Although $U_{1, \Phi}$ is not defined using an integral representation, it majorizes the integral on the right of 6 6.8), with equality for $|y| \leq h(|x|)$ and hence also for $|x|+|y| \leq h(0)$. This is all we need to apply the above argument with the function $W_{1}$. The strictness follows.

Now we turn to the optimality of the constants.

THEOREM 7.2.

(i) The constants $L_{p, q}, C_{p, q}$ given by (5.1) and (5.2) are the best possible even in the case $\mathcal{H}=\mathbb{R}$ and when $g$ is a \pm 1 transform of $f$. 
(ii) If

$$
\lim _{t \rightarrow \infty} \frac{\Phi(t+h(t))-\Phi(t)-\Phi^{\prime}(t) h(t)}{\exp \left(\Phi^{\prime}(t)\right)}=0,
$$

then the constant $L(\Phi)$ defined by 6.10 is the best possible even in the case $\mathcal{H}=\mathbb{R}$ and when $g$ is a \pm 1 transform of $f$.

The proof will be based on the construction of an appropriate example, which will work for (i) in the case $1 \leq p<q<2$, and for (ii) simultaneously. Suppose $h$ is the solution to $2.1 p_{p, q}$ for some $1 \leq p<q<2$ or is given by (6.4). Let $U, V$ denote $U_{p, q}, V_{p, q}$ or $U_{1, \Phi}, V_{1, \Phi}$, respectively. Furthermore, in the first case, assume that $\Phi(t)=t^{q}, t \in[0, \infty)$, and observe that $(7.2)$ is valid, which is a consequence of $h(t) / t \rightarrow 0$ as $t \rightarrow \infty$ (the latter being due to $h^{\prime}(t) \rightarrow 0$ as $\left.t \rightarrow \infty\right)$.

We start with the following auxiliary result.

Lemma 7.3. Assume $\mathcal{H}=\mathbb{R}$ and let $x>h(0)$ and $\delta \in(0, h(0))$. Then

$$
\begin{aligned}
U(x+\delta, \delta)= & \frac{\delta}{x+2 \delta-H(x+2 \delta)} U(H(x+2 \delta), x+2 \delta-H(x+2 \delta)) \\
& +\frac{x+\delta-H(x+2 \delta)}{x+2 \delta-H(x+2 \delta)} U(x+2 \delta, 0)
\end{aligned}
$$

and

$$
\begin{aligned}
U(x, 0)= & \frac{\delta}{x-H(x)+\delta} U(H(x), x-H(x)) \\
& +\frac{x-H(x)}{x-H(x)+\delta} U(x+\delta, \delta)+R(x, \delta),
\end{aligned}
$$

with $R(x, \delta)$ satisfying

$$
\lim _{\delta \rightarrow 0} \sup _{h(0) \leq x \leq T} \frac{R(x, \delta)}{\delta}=0
$$

for any $T>h(0)$.

Proof. The first equality follows immediately from the fact that for any $x \geq h(0)$, the function $t \mapsto U(x-t, t), t \in[0, x-H(x)]$, is linear. This property is also needed in the second part: we may write

$$
\begin{aligned}
\frac{R(x, \delta)}{\delta}= & \frac{-U(H(x), x-H(x))+U(x, 0)}{x-H(x)+\delta} \\
& +\frac{x-H(x)}{x-H(x)+\delta} \cdot \frac{U(x, 0)-U(x+\delta, \delta)}{\delta},
\end{aligned}
$$

and since $U_{y}(x, 0)=0$, we have

$$
U(H(x), x-H(x))-U(x, 0)=(H(x)-x) U_{x}(x, 0) .
$$


Therefore,

$$
\frac{R(x, \delta)}{\delta}=\frac{x-H(x)}{x-H(x)+\delta}\left(U_{x}(x, 0)-\frac{U(x+\delta, \delta)-U(x, 0)}{\delta}\right)
$$

and, by the mean value property,

$$
\begin{aligned}
\left|\frac{R(x, \delta)}{\delta}\right| & \leq\left|U_{x}(x, 0)-\frac{U(x+\delta, 0)-U(x, 0)}{\delta}-\frac{U(x+\delta, \delta)-U(x+\delta, 0)}{\delta}\right| \\
& \leq\left|U_{x}(x, 0)-U_{x}(\xi, 0)\right|+\left|U_{y}(x+\delta, \eta)\right|
\end{aligned}
$$

for some $\xi \in(x, x+\delta), \eta \in(0, \delta)$. Now it is easy to see that 7.5 is valid for any $T$ : this follows from the uniform continuity of $U_{x}(\cdot, 0)$ on $[h(0), T+1]$ and the uniform continuity of $U_{y}$ on $[h(0), T+1] \times[0, h(0)]$.

Now let us study the following example. Let $T>h(0)$ be a fixed (large) positive number. Let $N$ be an integer and $\delta$ be a positive number such that $T=h(0)+2 N \delta$. Eventually we will let $\delta$ go to 0 (and hence $N$ to $\infty$ ).

Consider a Markov martingale $(f, g)$, determined uniquely by the following conditions.

(i) It starts from $(h(0) / 2, h(0) / 2)$ almost surely.

(ii) From $(h(0) / 2, h(0) / 2)$ it moves either to $(h(0), 0)$, or to $(0, h(0))$.

(iii) For $h(0) \leq x<T$, the state $(x, 0)$ leads to $(H(x), h(H(x)))$ or to $(x+\delta,-\delta)$.

(iv) For $h(0) \leq x<T$, the state $(x+\delta,-\delta)$ leads to $(H(x+2 \delta)$, $-h(H(x+2 \delta)))$ or to $(x+2 \delta, 0)$.

(v) For $x \geq T$, the states $(x, 0)$ are absorbing.

(vi) All the states lying on the curves $y= \pm h(x)$ are absorbing.

Note that in (ii)-(iv) we do not need to specify the transity probabilities, as they are determined by the martingale property. Note that $g$ is a \pm 1 transform of $f$.

From the proof of 1.5 we know that the sequence $\left(\mathbb{E} U\left(f_{n}, g_{n}\right)\right)$ is nonincreasing. The lemma below states that it is almost constant.

LEMma 7.4. We have

$$
\mathbb{E} U\left(f_{2 N}, g_{2 N}\right) \geq U(h(0) / 2, h(0) / 2)-N \sup _{h(0) \leq x \leq T}|R(x, \delta)| .
$$

Proof. We start from the observation that, by Lemma 7.3,

$$
\mathbb{E} U\left(f_{2 n}, g_{2 n}\right)=\mathbb{E} U\left(f_{2 n+1}, g_{2 n+1}\right)
$$

for $n=0,1, \ldots, N$, and

$$
\mathbb{E} U\left(f_{2 n+1}, g_{2 n+1}\right)=\mathbb{E} U\left(f_{2 n+2}, g_{2 n+2}\right)+R\left(x_{2 n+1}, \delta\right)
$$


for $n=0,1, \ldots, N-1$, where we have set $x_{n}=h(0)+(n-1) \delta$. This gives

$$
U(h(0) / 2, h(0) / 2)=\mathbb{E} U\left(f_{0}, g_{0}\right)=\mathbb{E} U\left(f_{2 N}, g_{2 N}\right)+\sum_{n=0}^{N-1} R\left(x_{2 n+1}, \delta\right),
$$

and the estimate follows.

It is easy to see from conditions (iii) and (iv) that the process $\left(f_{n}, g_{n}\right)$ moves from $(x, 0)$ to $(x+2 \delta, 0)$ in two steps with probability

$$
\left(1-\frac{\delta}{x-H(x)+\delta}\right)\left(1-\frac{\delta}{x+2 \delta-H(x+2 \delta)}\right) .
$$

Hence the probability $p_{\delta}$ that $\left(f_{n}, g_{n}\right)$ ever reaches $(T, 0)$ equals

$$
\frac{1}{2} \prod_{n=0}^{N-1}\left(1-\frac{\delta}{x_{2 n+1}-H\left(x_{2 n+1}\right)+\delta}\right)\left(1-\frac{\delta}{x_{2 n+1}+2 \delta-H\left(x_{2 n+1}+2 \delta\right)}\right) .
$$

In the next lemma we study the limit behavior of $p_{\delta}$ as $\delta \rightarrow 0$.

LEMMA 7.5. We have

$$
\lim _{\delta \rightarrow 0} p_{\delta}=\frac{1}{2} \exp \left(-\Phi^{\prime}(H(T))\right) .
$$

Proof. We find that

$$
\frac{1}{2} \exp \left[\sum_{n=0}^{N-1}\left(\frac{\delta}{x_{2 n+1}-H\left(x_{2 n+1}\right)+\delta}+\frac{\delta}{x_{2 n+1}+2 \delta-H\left(x_{2 n+1}+2 \delta\right)}\right)\right] \cdot p_{\delta}^{-1}
$$

converges to 1 as $\delta \rightarrow 0$, and the Riemann sum

$$
\sum_{n=0}^{N-1}\left(\frac{\delta}{x_{2 n+1}-H\left(x_{2 n+1}\right)+\delta}+\frac{\delta}{x_{2 n+1}+2 \delta-H\left(x_{2 n+1}+2 \delta\right)}\right)
$$

converges, as $\delta \rightarrow 0$, to

$$
-\int_{h(0)}^{T} \frac{1}{x-H(x)} d x=-\int_{h(0)}^{T} \frac{1}{h(H(x))} d x .
$$

Substitution $y=H(x)$ (so $x=y+h(y)$ ) transforms the integral into

$$
-\int_{0}^{H(T)} \frac{1+h^{\prime}(y)}{h(y)} d y=-\int_{0}^{H(T)} \Phi^{\prime \prime}(y) d y=-\Phi^{\prime}(H(T)) .
$$

This yields the claim.

Proof of Theorem 7.2. For convenience, let us divide the proof into a few steps.

STEP 1: Sharpness of 1.5) for $1 \leq p<q<2$ and sharpness of 6.3). Note that on the set $\{(x, y): y= \pm h(x)\}$ the functions $U$ and $V$ coincide. 
The variable $\left(f_{2 N}, g_{2 N}\right)$ belongs to this set unless $\left(f_{2 N}, g_{2 N}\right)=(T, 0)$, which occurs with probability $p_{\delta}$. Hence we may write

$$
\begin{aligned}
& \mathbb{E} V\left(f_{2 N}, g_{2 N}\right)=\mathbb{E} U\left(f_{2 N}, g_{2 N}\right)+(V(T, 0)-U(T, 0)) p_{\delta} \\
& \quad \geq U(h(0) / 2, h(0) / 2)-N \sup _{h(0) \leq x \leq T}|R(x, \delta)|+(V(T, 0)-U(T, 0)) p_{\delta},
\end{aligned}
$$

where we have used Lemma 7.4. Now let $\delta \rightarrow 0$. By 7.5 and the equality $T=h(0)+2 N \delta$, we have

$$
N \sup _{h(0) \leq x \leq T}|R(x, \delta)|=\frac{T-h(0)}{2 \delta} \sup _{h(0) \leq x \leq T}|R(x, \delta)| \rightarrow 0 .
$$

Furthermore, by Lemmas 4.3 and 7.5 , we have

$$
\begin{aligned}
0 \leq(V(T, 0)-U(T, 0)) p_{\delta} & \\
& \rightarrow-\frac{\Phi(T)-\Phi(H(T))-\Phi^{\prime}(H(T))(T-H(T))}{2 \exp \left(\Phi^{\prime}(H(T))\right)} .
\end{aligned}
$$

Now we proceed as follows. By 7.2 , for a fixed $\varepsilon>0$ we may choose $T$ such that the expression appearing on the right is greater than $-\varepsilon$. Keeping this $T$ fixed, we use (7.6) and (7.7) to choose $\delta>0$ for which

$$
\mathbb{E}\left|g_{2 N}\right|-\mathbb{E} \Phi\left(\left|f_{2 N}\right|\right)=\mathbb{E} V\left(f_{2 N}, g_{2 N}\right) \geq U(h(0) / 2, h(0) / 2)-3 \varepsilon .
$$

However, $U(h(0) / 2, h(0) / 2)$ equals $L_{p, q}$ or $L(\Phi)$, depending on the choice of $h$ in the example; hence, as $\varepsilon$ is arbitrary, these constants are best possible.

STEP 2: Optimality of $C_{p, q}, 1 \leq p<q<\infty$. If $1 \leq p \leq 2 \leq q<\infty$, then, obviously, $C_{p, q}=1$ is the best possible (take $f=g \equiv 1$ ). Now, by duality and Remark 5.2(ii), it suffices to deal with the case $1 \leq p<q<2$. For fixed $\varepsilon>0$, let $f^{\varepsilon}, g^{\varepsilon}$ be real martingales such that $g^{\varepsilon}$ is a \pm 1 transform of $f^{\varepsilon}$ and

$$
\left\|g^{\varepsilon}\right\|_{p}^{p}>\left\|f^{\varepsilon}\right\|_{q}^{q}+L_{p, q}-\varepsilon .
$$

Let

$$
\lambda=\left(\frac{p}{q-p} \cdot \frac{L_{p, q}-\varepsilon}{\|f\|_{q}^{q}}\right)^{(q-p) / q} .
$$

The martingale $\tilde{g}=g^{\varepsilon} \cdot \lambda^{1 /(q-p)}$ is a \pm 1 transform of $\tilde{f}=f^{\varepsilon} \cdot \lambda^{1 /(q-p)}$. Multiply both sides of $(7.8)$ by $\lambda^{p /(q-p)}$ to obtain

$$
\|\tilde{g}\|_{p}^{p}>\left[\left(L_{p, q}-\varepsilon\right)^{(q-p) / p q} \cdot\left(\frac{q-p}{p}\right)^{1 / q}\left(\frac{q}{q-p}\right)^{1 / p}\right]^{p}\|\tilde{f}\|_{q}^{p} .
$$

It is clear that the expression in the square brackets can be made arbitrarily close to $C_{p, q}$. This implies $C_{p, q}$ cannot be replaced by a smaller constant in 1.4.

SteP 3: Sharpness of 1.5) for $2<p<q<\infty$. This is an immediate consequence of Step 2. If $L_{p, q}$ were not optimal for some $p, q$, then $C_{p, q}$ 
would not be the best either, as can be easily seen by examining the proof of Theorem 5.1.

We conclude the paper by proving that the $\log \log$ inequality established in the previous section is sharp. Let $\Phi(t)=t \log (t+1)+K t \log \log (t+e)$ and let $h$ be given by 6.4 .

Lemma 7.6. If $K>1$, then the condition (7.2) is valid.

Proof. By de l'Hospital's rule, it suffices to show that

$$
\lim _{t \rightarrow \infty} \frac{\Phi^{\prime}(t+h(t))\left(1+h^{\prime}(t)\right)-\Phi^{\prime}(t)\left(1+h^{\prime}(t)\right)-\Phi^{\prime \prime}(t) h(t)}{\exp \left(\Phi^{\prime}(t)\right) \Phi^{\prime \prime}(t)}=0 .
$$

Since $1+h^{\prime}(t)=h(t) \Phi^{\prime \prime}(t)$, this can be rewritten in the form

$$
\lim _{t \rightarrow \infty} \frac{\left[\Phi^{\prime}(t+h(t))-\Phi^{\prime}(t)-1\right] h(t)}{\exp \left(\Phi^{\prime}(t)\right)}=0 .
$$

As $h(t) / \exp \left(\Phi^{\prime}(t)\right)=\int_{t}^{\infty} \exp \left(-\Phi^{\prime}(s)\right) d s$ tends to 0 , we must show that

$$
\lim _{t \rightarrow \infty}\left(\Phi^{\prime}(t+h(t))-\Phi^{\prime}(t)\right) \int_{t}^{\infty} \exp \left(-\Phi^{\prime}(s)\right) d s=0 .
$$

We have, by 6.12,

$$
\begin{aligned}
\int_{t}^{\infty} \exp \left(-\Phi^{\prime}(s)\right) d s & \leq \int_{t}^{\infty} \frac{d s}{(s+1)(\log (s+e))^{K}} \\
& \leq \int_{t}^{\infty} \frac{e d s}{(s+e)(\log (s+e))^{K}}=\frac{e(\log (t+e))^{1-K}}{K-1}
\end{aligned}
$$

Furthermore, as

$$
\Phi^{\prime}(t) \leq \log (t+1)+K \log \log (t+e)+K+1,
$$

we have

$$
\begin{aligned}
h(t) & \leq \exp \left(\Phi^{\prime}(t)\right) \cdot \frac{e(\log (t+e))^{1-K}}{K-1} \\
& \leq e^{K+1}(t+1)(\log (t+e))^{K} \cdot \frac{e(\log (t+e))^{1-K}}{K-1} \\
& =\frac{e^{K+2}}{K-1}(t+1) \log (t+e) .
\end{aligned}
$$

Therefore, by 6.12,

$$
\begin{aligned}
0 \leq \Phi^{\prime}(t+h(t))-\Phi^{\prime}(t) \leq & K \log \log (t+h(t)+e)-K \log \log (t+e) \\
& +\log (t+h(t)+1)-\log (t+1)+(K+1) .
\end{aligned}
$$


Now, by 7.10 and 7.11 ,

$$
\begin{aligned}
(\log (t+h(t)+1) & -\log (t+1)) \int_{t}^{\infty} \exp \left(-\Phi^{\prime}(s)\right) d s \\
& \leq \log \left(\frac{e^{K+2}}{K-1} \log (t+e)+1\right) \frac{e(\log (t+e))^{1-K}}{K-1} \rightarrow 0
\end{aligned}
$$

as $t \rightarrow \infty$. Furthermore, again by (7.11), we may write

$$
\begin{aligned}
\log \log (t+h(t)+e)-\log & \log (t+e) \\
& =\log \left(1+\frac{\log (t+h(t)+e)-\log (t+e)}{\log (t+e)}\right) \\
\leq & \log \left(1+\frac{\log \left(\frac{e^{K+2}}{K-1} \log (t+e)\right)}{\log (t+e)}\right) \rightarrow 0
\end{aligned}
$$

as $t \rightarrow 0$. This yields 7.9 and hence 7.2 .

Acknowledgments. The author would like to thank the anonymous referee for a careful reading of the first version of the manuscript and for many remarks and suggestions which greatly improved the presentation. The research was partially supported by MNiSW Grant N N201 364436 and Foundation for Polish Science.

\section{References}

[BW1] R. Bañuelos and G. Wang, Sharp inequalities for martingales with applications to the Beurling-Ahlfors and Riesz transformations, Duke Math. J. 80 (1995), $575-600$.

[BW2] -, Davis's inequality for orthogonal martingales under differential subordination, Michigan Math. J. 47 (2000), 109-124.

[Bu1] D. L. Burkholder, A geometrical characterization of Banach spaces in which martingale difference sequences are unconditional, Ann. Probab. 9 (1981), 997-1011.

[Bu2] -, Boundary value problems and sharp inequalities for martingale transforms, Ann. Probab. 12 (1984), 647-702.

[Bu3] - Differential subordination of harmonic functions and martingales, in: Harmonic Analysis and Partial Differential Equations (El Escorial, 1987), Lecture Notes in Math. 1384, Springer, 1989, 1-23.

[Bu4] - Explorations in martingale theory and its applications, in: École d'Été de Probabilités de Saint-Flour XIX—1989, Lecture Notes in Math. 1464, Springer, Berlin, 1991, 1-66.

[Bu5] -, Strong differential subordination and stochastic integration, Ann. Probab. 22 (1994), 995-1025.

[Bu6] -, Some extremal problems in martingale theory and harmonic analysis, in: Harmonic Analysis and Partial Differential Equations (Chicago, IL, 1996), Chicago Lectures in Math., Univ. Chicago Press, Chicago, IL, 1999, 99-115. 
[C1] C. Choi, A submartingale inequality, Proc. Amer. Math. Soc. 124 (1996), 25492553.

[C2] -, An inequality of subharmonic functions, J. Korean Math. Soc. 34 (1997), $543-551$.

[C3] - A weak-type submartingale inequality, Kobe J. Math. 14 (1997), 109-121.

[C4] - A weak-type inequality for differentially subordinate harmonic functions, Trans. Amer. Math. Soc. 350 (1998), 2687-2696.

[Ha1] W. Hammack, Sharp inequalities for the distribution of a stochastic integral in which the integrator is a bounded submartingale, Ann. Probab. 23 (1995), 223235 .

[Ha2] - Sharp maximal inequalities for stochastic integrals in which the integrator is a submartingale, Proc. Amer. Math. Soc. 124 (1996), 931-938.

[O1] A. Osękowski, Sharp norm inequalities for martingales and their differential subordinates, Bull. Polish Acad. Sci. Math. 55 (2007), 373-385.

[O2] - Sharp LlogL inequalities for differentially subordinated martingales, Illinois J. Math. 52 (2008), 745-756.

[O3] - Sharp norm inequalities for stochastic integrals in which the integrator is a nonnegative supermartingale, Probab. Math. Statist. 29 (2009), 29-42.

[O4] —, Sharp weak type inequalities for differentially subordinated martingales, Bernoulli 15 (2009), 871-897.

[S] Y. Suh, A sharp weak type $(p, p)$ inequality $(p>2)$ for martingale transforms and other subordinate martingales, Trans. Amer. Math. Soc. 357 (2005), 1545-1564.

[W] G. Wang, Differential subordination and strong differential subordination for continuous time martingales and related sharp inequalities, Ann. Probab. 23 (1995), $522-551$.

Adam Osękowski

Department of Mathematics, Informatics and Mechanics

University of Warsaw

Banacha 2

02-097 Warszawa, Poland

E-mail: ados@mimuw.edu.pl

Received March 20, 2009

Revised version October 12, 2010 